Purdue University

Purdue e-Pubs

\title{
An experimental investigation of the effect of thermal coupling between parallel microchannels undergoing boiling on the Ledinegg instability-induced flow maldistribution
}

\author{
A. Miglani \\ Purdue University
}

J. A. Weibel

Purdue University, jaweibel@purdue.edu

S V. Garimella

University of Vermont, sureshg@purdue.edu

Follow this and additional works at: https://docs.lib.purdue.edu/coolingpubs

Miglani, A.; Weibel, J. A.; and Garimella, S V., "An experimental investigation of the effect of thermal coupling between parallel microchannels undergoing boiling on the Ledinegg instability-induced flow maldistribution" (2021). CTRC Research Publications. Paper 374.

http://dx.doi.org/https://doi.org/10.1016/j.ijmultiphaseflow.2020.103536

This document has been made available through Purdue e-Pubs, a service of the Purdue University Libraries.

Please contact epubs@purdue.edu for additional information. 


\title{
An experimental investigation of the effect of thermal coupling between parallel microchannels undergoing boiling on the Ledinegg instability-induced flow maldistribution
}

\author{
Ankur Miglani ${ }^{\mathrm{a}, \mathrm{b}}$, Justin A. Weibe ${ }^{\mathrm{a}^{*}}$ and Suresh V. Garimella $\mathrm{a}^{\mathrm{a}^{* *}}$ \\ ${ }^{a}$ School of Mechanical Engineering, Purdue University, West Lafayette, IN 47907 USA \\ ${ }^{b}$ Department of Mechanical Engineering, Indian Institute of Technology, Indore, Madhya Pradesh 453552, India
}

\begin{abstract}
Two-phase flow boiling is susceptible to the Ledinegg instability, which can result in non-uniform flow distribution between parallel channels and thereby adversely impact the heat transfer performance. This study experimentally assesses the effect of thermal coupling between the parallel channels on flow maldistribution caused by the Ledinegg instability and compares the results to our prior theoretical predictions. A system with two parallel microchannels is investigated using water as the working fluid. The channels are hydrodynamically connected via common inlet/outlet plenums and supplied with a constant total flow rate. The channels are uniformly subjected to the same input power (which is increased in steps). Two separate configurations are evaluated to assess drastically different levels of thermal coupling between the channels, namely thermally isolated and thermally coupled channels. Synchronized measurements of the flow rate in each individual channel, wall temperature, and pressure drop are performed along with flow visualization to compare the thermal-hydraulic characteristics of these two configurations. Thermal coupling is shown to reduce the wall temperature difference between the channels and dampen flow maldistribution. Specifically, the range of input power over which flow
\end{abstract}

\footnotetext{
* Corresponding author: jaweibel@purdue.edu

${ }^{* *}$ Currently President, University of Vermont
} 
maldistribution occurs is noticeably smaller and the maximum severity of flow maldistribution is reduced in thermally coupled channels. The data provide a quantitative account of the effect of lateral thermal coupling in moderating flow maldistribution, which is corroborated by comparison to predictions from our two-phase flow distribution model. This combined experimental and theoretical evidence demonstrates that, under extreme conditions when one channel is significantly starved of flow rate and risks dryout, channel-to-channel thermal coupling can redistribute the heat load from the flow-starved channel to the channel with excess flow. Due to such a possibility of heat redistribution, the coupled channels are significantly less prone to flow maldistribution compared to thermally isolated channels.

Keywords: Flow boiling; Ledinegg instability; maldistribution; parallel microchannels; thermal coupling

\section{Nomenclature}

$\begin{array}{ll}A_{\text {wall }} & \text { total area of the walls of a single channel }\left(2 H_{c} L_{h}+W_{c}\right) \\ A_{b} & \text { cross-sectional area of the channel block }\left(H_{b} W_{b}-H_{c} W_{c}\right) \\ C_{l a t} & \text { lateral thermal conductance } \\ H & \text { height } \\ I & \text { electric current } \\ k & \text { thermal conductivity } \\ L & \text { length } \\ P & \text { power applied to each channel block } \\ P_{\text {in }} & \text { heating power going into the channel } \\ P_{\text {loss }} & \text { power loss to ambient } \\ P_{T} & \text { total power applied to the test section }(2 P) \\ P_{T, \text { in }} & \text { total power going into the channels }\left(2 P_{\text {in }}\right) \\ p_{\text {out }} & \text { outlet pressure }\end{array}$




$\begin{array}{ll}\Delta p_{u h} & \text { pressure drop across the unheated channel length } \\ \Delta p_{h} & \text { pressure drop across the heated channel length } \\ \Delta p_{o} & \text { overall pressure drop across the channels } \\ Q & \text { volumetric flow rate } \\ q_{\text {in }}^{\prime \prime} & \text { heat flux into the channel }\left(q_{\text {in }}^{\prime \prime}=P_{\text {in }} / A_{\text {wall }}\right) \\ S & \text { pitch } \\ T & \text { temperature } \\ T_{f l, i n} & \text { inlet fluid temperature } \\ T_{f l, o u t} & \text { outlet fluid temperature } \\ T_{\text {sat }} & \text { saturation fluid temperature } \\ T_{\text {wall,i }} & \text { wall temperature of the } i^{t h} \text { channel } \\ V & \text { voltage } \\ W & \text { width } \\ y & \text { vertical coordinate } \\ Z & \text { streamwise coordinate }\end{array}$

$\begin{array}{ll}\text { Subscripts } \\ \text { air } & \text { air gap } \\ b & \text { channel block } \\ c & \text { channel } \\ \text { exp } & \text { experiment } \\ h & \text { heated } \\ i & \text { channel index ( } i=1 \text { or } 2) \\ \text { mod } & \text { model } \\ \text { wall } & \text { channel wall }\end{array}$

Greek Letters

$\varepsilon_{i} \quad$ fraction of the total flow rate going into channel $i\left(Q_{i} / Q_{T}\right)$ 


\section{Introduction}

Over the last three decades, microscale two-phase heat sinks and cold plates have been explored for low-pumping-power cooling of power-dense electronics in applications such as data centers [1-2], traction inverters of hybrid and electric vehicles [3-4], and radars [5]. The coolant flow is typically routed through multiple parallel channels to maximize the heat transfer area. Such two-phase flow cooling strategies are attractive because they improve the heat transfer performance and reduce the working temperatures and temperature gradients by utilizing the latent heat of vaporization, while requiring a lower coolant flow rate (compared to single-phase cooling). However, boiling flows are inherently prone to instabilities which may adversely impact the heat sink performance, and in some cases, even lead to a premature dry-out at heat fluxes lower than the predicted critical heat flux $(\mathrm{CHF})[6,7]$. Flow boiling instabilities are commonly categorized as either dynamic or static instabilities [8-12]. Static instabilities, the focus of the current work, occur when a small disturbance causes the system to suddenly transition to a new stable operating point that is significantly different from the initial condition.

One static instability of significant interest is the Ledinegg instability [13] because it induces flow maldistribution even under steady and uniform heating conditions. The Ledinegg instability is a consequence of the non-monotonic channel demand curve (channel pressure drop versus flow rate) and the supply pump curve in flow boiling systems. For a single channel, the Ledinegg instability occurs when the slope of the supply pump curve is greater than that of the channel demand curve and is characterized by a drastic reduction in the flow through the channel. In a system with multiple parallel channels, the Ledinegg instability results in non-uniform flow distribution between the channels. This is detrimental to heat sink performance because the channels that are starved of flow relative to a uniform flow distribution may undergo dry-out. This 
may trigger an apparent premature critical heat flux and limit the heat sink performance predictability. Several methods have been proposed to dampen the flow maldistribution caused by the Ledinegg instability, including inlet orifices and throttle valves [7, 14-15], active control of the flow at the channel inlet through pumps and valves [16-18], and increasing the system pressure [19]. However, these measures have an associated penalty of significantly increasing either the system complexity or pressure drop (i.e., pumping power), thereby reducing the attractiveness of two-phase heat sinks.

Several studies have characterized the flow maldistribution caused by the Ledinegg instability. For instance, Akagawa et al. [20] investigated the flow maldistribution in multiple large evaporator tubes ( $4 \mathrm{~mm}$ inner diameter; $40 \mathrm{~m}$ long). They obtained the demand curves for each tube undergoing flow boiling and measured the flow rate distribution in a system with up to three parallel tubes. It was demonstrated that the flow rate distribution in a multiple-tube system could be estimated from the individual tube load curves. A modeling approach was developed to generate a stability criterion by performing a Laplace transformation on the linearized momentum and continuity equations for a system of parallel tubes, which yielded predictions consistent with their experimental observations. Minzer et al. [21-22] investigated the flow distribution behavior in a system with two heated parallel tubes ( $5 \mathrm{~mm}$ inner diameter; $6 \mathrm{~m}$ long). In these studies, the flow rate measurements in individual tubes were obtained either through a pressure drop element (such as an inlet restrictor, throttle valve, or flow meter) at the inlet of each channel or by collecting and measuring the fluid volume exiting each channel. Notably, these studies also focused on large separate tubes that are thermally isolated from each other, differing drastically from parallel microchannel heat sinks used in electronics cooling applications, where channels are thermally connected via a common substrate. Kingston et al. [23] experimentally investigated the 
temperature non-uniformity caused by the Ledinegg instability in two thermally isolated, parallel channels. Two cylindrical microchannels were uniformly subjected to the same power which was increased in steps. With increasing power, when boiling occurred in one of the channels, the Ledinegg instability triggered a temperature difference between the channels, which grew with increasing power. The wall temperature and heat flux measurements were used to attribute the observed behavior to the increasing severity of flow maldistribution between the channels. In our recent study [24], we extended the approach presented in Ref. [23] by enabling a direct measurement of the flow rate in each channel synchronized with the wall temperature and overall pressure drop measurements to characterize the thermal and hydrodynamic effects of the Ledinegg instability on thermally isolated channels. It was demonstrated that once flow maldistribution is triggered by the Ledinegg instability, its severity grows with increasing power. This causes the temperature of the flow-starved channel to increase continuously and the wall temperature difference to grow with increasing power.

The severity of the flow maldistribution encountered in the studies reviewed above, which considered a special case of thermally isolated parallel channels, is exaggerated compared to experimental experience [25-28]. In actual microchannel heat sinks, however, the high thermal conductivity of the fins and substrate results in strong lateral thermal coupling between neighboring channels and allows for heat redistribution. This contrasts with most past studies on the Ledinegg instability which used parallel pipes that are physically isolated from one another [20-22].

A few studies have considered the effect of lateral thermal coupling on dampening the flow maldistribution between parallel channels. Flynn et al. [25, 29] studied the thermal implications of flow maldistribution between two parallel microchannels (etched in a silicon substrate) that were 
either thermally coupled or thermally isolated. The heat input to each of the channels could be varied independently and they were tested under both uniform and non-uniform heating conditions. In the thermally isolated configuration, the channel subjected to a higher heat load underwent boiling, while the other channel remained a single-phase liquid, leading to a noticeable channelto-channel temperature difference. Based on the observed temperature difference it was inferred that flow maldistribution existed between the channels (as no direct measurement could be made of the flow rate to each channel). In contrast, in the thermally coupled configuration, the flow in both channels either remained a single-phase liquid or they both underwent boiling, even under severe non-uniform heating. No significant wall temperature difference existed between the channels, from which it was inferred that the flow distribution was uniform. Van Oevelen et al. [30] were the first to theroretically investigate the effect of channel-to-channel thermal coupling on flow distribution by accounting for axial and lateral wall conduction. They predicted that increasing the strength of thermal coupling between the channels reduced the severity of flow maldistribution via redistribution of heat from the flow-starved channel to the adjacent channels with excess flow. Additionally, they identified a threshold heat flux below which the flow maldistribution can be completely suppressed by this mechanism. This threshold heat flux increases with an increase in thermal coupling and eventually asymptotes to a constant value in the limit of very strong thermal coupling. These past efforts have identified the critical implications of channel-to-channel thermal coupling on determining the Ledinegg-instability-induced flow maldistribution in boiling parallel microchannels. However, experimental characterization of the Ledinegg instability in thermally coupled channels has been lacking. In particular, the measurement of flow rates in individual channels is needed to corroborate past theoretical predictions. Consideration of the role of lateral thermal coupling in moderating flow 
maldistribution will allow other mitigation techniques such as inlet throttling to be more sparingly employed.

In this study, the experiments are designed to incorporate and study the effect of thermal wall conductance on the flow distribution between two parallel microchannels undergoing boiling. Experiments are performed under thermally isolated and thermally coupled conditions that represent two extreme levels of thermal connectivity between the channels. Comparison between the thermally isolated case and the thermally coupled case allows any change in the flow distribution behavior, as well as the measured wall temperature difference and overall pressure drop, to be attributed solely to the mechanism of heat transfer between the channels. Synchronized measurements of the wall temperature, the flow rate in each channel, and the overall pressure drop are presented to illustrate the differences between the isolated and coupled cases. The experiments demonstrate that channel-to-channel thermal coupling (via heat conduction through the wall) plays a critical role in moderating flow maldistribution between the channels by allowing redistribution of the heat flux. In the last section, these experimental results are directly compared with predictions from our two-phase flow distribution model $[30,31]$ and are shown to have an excellent match, thereby confirming the mitigating influence of thermal coupling on flow maldistribution.

\section{Experimental methods}

\subsection{Test facility}

A photograph of the custom-built experimental facility for investigating the effect of thermal coupling between boiling parallel microchannels on the flow maldistribution caused by the Ledinegg instability is shown in Fig. 1. The flow loop components, aside from the test section, are identical to the facility presented in Ref. [24] and are summarized here. A magnetically- 
coupled gear pump (GA V21, Micropump) circulates the degassed DI water through a closed loop. The water flow rate is measured using a liquid flow meter (LC-10CCM, Alicat; accuracy of $\pm 1 \%$ full scale), and tuned to a constant set-point value by controlling a metering valve placed in the recirculation line. The fluid temperature at the test section inlet is controlled using an inline heater $(120 \mathrm{~V} \mathrm{AC}, 468 \mathrm{~W})$. The inlet and outlet fluid temperatures are measured using calibrated Ttype thermocouples (TMTSS-020E-6, Omega Engineering; $\pm 0.3^{\circ} \mathrm{C}$ ) located immediately upstream and downstream of the test section, respectively.

Pressure drop across the test-section $\left(\Delta p_{o}\right)$ is measured with a differential pressure transducer (PX154-005DI Wet-Wet, Omega; 0 - $1250 \mathrm{~Pa} ; \pm 2 \%$ full scale). The pressure sensing ports for the overall pressure drop measurement are in the test-section inlet and outlet plenums. Fluid exiting the test section returns to the reservoir and then enters a liquid-to-air heat exchanger where it is cooled before entering the pump inlet. The reservoir contains excess fluid and has an adjustable volume which allows the system pressure to be set to a desired value of $104.4 \mathrm{kPa}$, which is measured at the test-section outlet using an absolute pressure transducer (PX309030G5V, Omega Engineering; $\pm 1 \%$ ). The entire experimental facility is mounted on a vibrationisolated optical table (VIS3672-PG2-325A, Newport Corp.) to ensure that external vibrations are not transmitted to the components. 


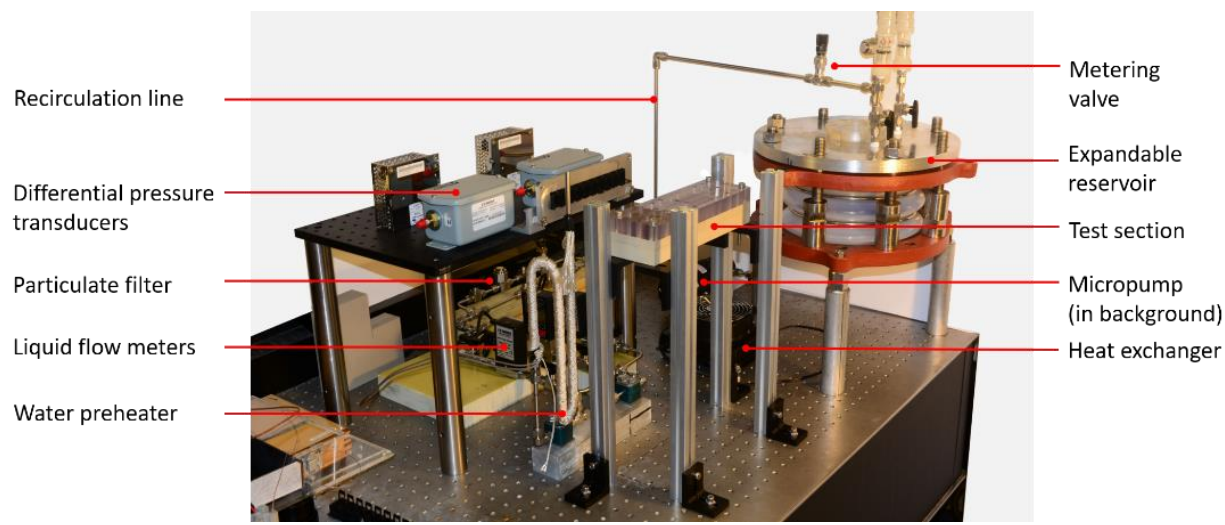

Fig. 1. A photograph of the experimental test facility with key components labeled. [1.5 columns]

\subsection{Test section}

The test section used in this study is modified from Ref. [24] to allow operation in both thermally isolated and thermally coupled configurations. A quarter cut-away isometric view of the test-section assembly is shown in Fig. 2. It consists of three main components: a bottom PEEK plate, a middle portion with parallel microchannels, and a polycarbonate cover plate to enable flow visualization from above. These three components are stacked vertically and then bolted together. The middle portion comprises three sections in the flow direction: an upstream unheated section, the heated channel section (which differs between the isolated and coupled cases), and a downstream unheated section. The flow enters the inlet plenum in the upstream section and divides into two parallel microchannels, each with a cross-section of $1 \mathrm{~mm} \times 1 \mathrm{~mm}$. After traversing the entire channel length, the flow exits the test section via the outlet plenum in the downstream unheated section. 


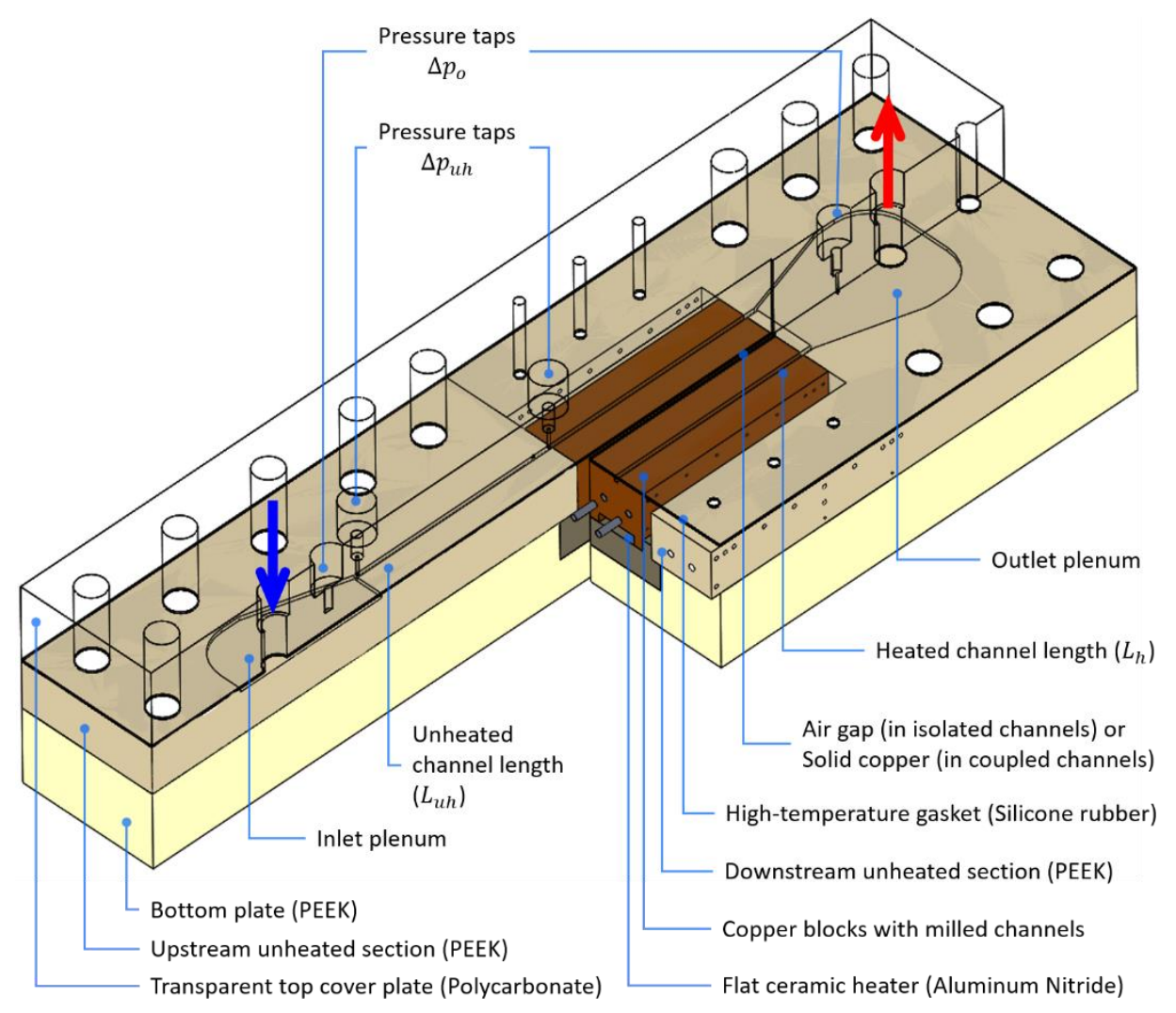

Fig. 2. A quarter cut-away isometric view of the test-section assembly drawing, with important components and the flow inlet and outlet pathways indicated. An air gap is maintained between the channel blocks in the thermally isolated configuration, whereas it is replaced by solid copper in the thermally coupled configuration. [1.5 columns]

The total channel length is divided into two equal parts in the flow direction. The first unheated half of the channel length $\left(L_{u h}=55 \mathrm{~mm}\right)$ lies in the upstream unheated section and is used as a flow rate sensor. Throughout this channel length, the fluid is always single-phase liquid. The flow rate is measured individually in the unheated section of each channel using differential pressure transducers (0-249 Pa PX154-001DI Wet-Wet type, Omega Engineering; $\pm 2 \%$ full scale), as shown in Fig. 2. Due to the low operating pressures (0 - $249 \mathrm{~Pa})$ of the transducer, the output response is sensitive to transient events such as bubble nucleation. Therefore, pressure snubbers 
(PS-8E, Omega) are installed at the pressure-sensing ports of the transducers to suppress fluctuations in the output signal and enable the flow rate in each channel to be measured accurately. Transparent tubing is used to connect the pressure taps on the test-section with the transducer ports (pressure sensing lines) to visually detect trapped air bubbles or vapor pockets, which can be removed by bleeding the line prior to testing. The heated second half of the channel length $\left(L_{h}=\right.$ $55 \mathrm{~mm}$ ) lies in the heated section in which the wall temperature measurements and flow visualization are performed.

The copper blocks that form the heated section consist of two parallel microchannels situated in close proximity, as shown in Fig. 3. The geometric parameters of the heated section are presented Table 1. Both thermally isolated (Fig. 3a) and thermally coupled (Fig. 3b) configurations are considered. In the thermally isolated configuration (Fig. 3a), the channels are milled into individual copper blocks that are separated from each other by a $1 \mathrm{~mm}$ thick air gap, significantly increasing the lateral channel-to-channel thermal resistance. This air gap between the channels runs through the entire height of the test-section assembly (Fig. 2). In the thermally coupled configuration (Fig. 3b), the channels are milled into a single copper block that allows channel-tochannel heat redistribution via conduction through the solid copper. The overall width of the heated section $(31 \mathrm{~mm})$ and the channel-to-channel pitch $(16 \mathrm{~mm})$ are identical for both the isolated and coupled cases. A quantitative measure of the lateral thermal coupling between the channels for the isolated versus coupled configurations, which are found to differ by two orders of magnitude, is provided later in Section 3.2. It is important to note that, aside from the air gap, the thermally isolated and coupled flow configurations are identical, allowing any effect on the flow maldistribution between the parallel channels to be attributed solely to the differing levels of lateral thermal coupling. 
In both isolated and coupled configurations, two adjustable power supplies (XG 50A-60V, Sorensen) are used to supply power to the heated channel section via two separate aluminum nitride heaters (582 W CER-1-01-00003, Watlow) that are mounted in rectangular recesses in the bottom of the channel blocks. Six thermocouples (TMTSS-020E-6, Omega Engineering) are used to measure the temperature along the block length and height. The locations of these thermocouple ports are marked by red arrows in Fig. 3(b). However, due to their high thermal conductivity (copper) and thick cross-section, the channel blocks attain a near-uniform temperature at steady state in all experiments. Therefore, all thermocouple readings are equal within the sensor accuracy and a single channel wall temperature is reported.

As described in Ref. [24], differential pressures, the overall pressure drop, channel wall temperatures, and power to each channel are recorded using a data acquisition (DAQ) unit (34970A, Agilent) at a rate of one sample every $15 \mathrm{~s}$. The total power applied to each channel (including the power loss to the ambient) is quantified by measuring the voltage drop and current flow through shunt resistors (HA 5 100, Empro). Flow is visualized from the top using a highspeed camera (VEO710L, Phantom) coupled with a macro lens (Makro-Planar T*2/100, Zeiss), with the lens focused on the top surface of the channel blocks in the field of view. The field of view $(1000 \times 620$ pixels) covers approximately $90 \%$ of the heated channel length. It is uniformly top-lit using a fiber-optic light source (Titan 300, Sunoptic Technologies) to aid visualization. The images are acquired at $200 \mathrm{fps}$ (exposure time of $5000 \mu \mathrm{s}$ ) at an optical resolution of $50 \mu \mathrm{m} / \mathrm{pixel}$. 
(a)

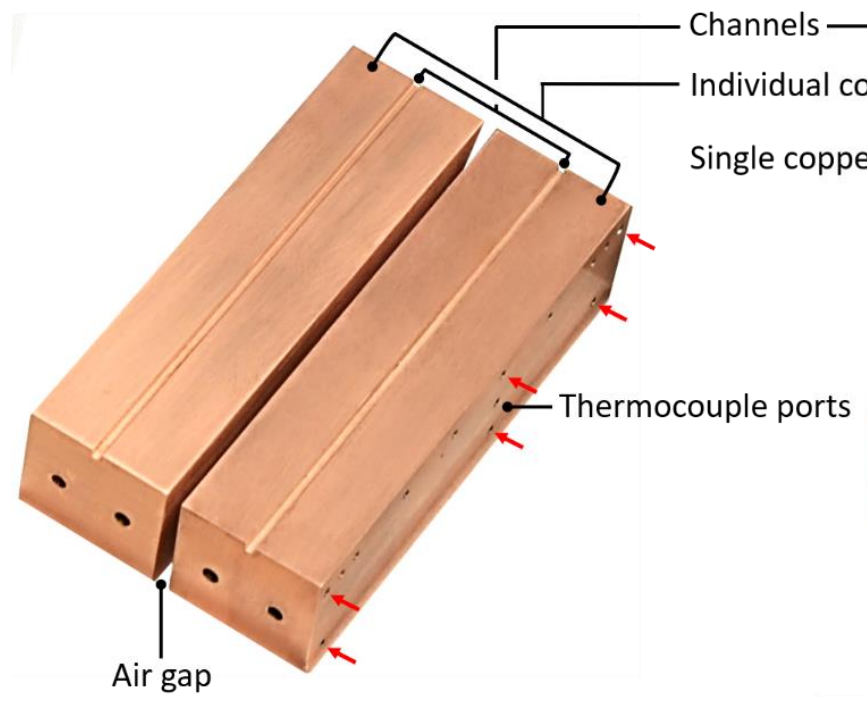

Thermally isolated channels (b)

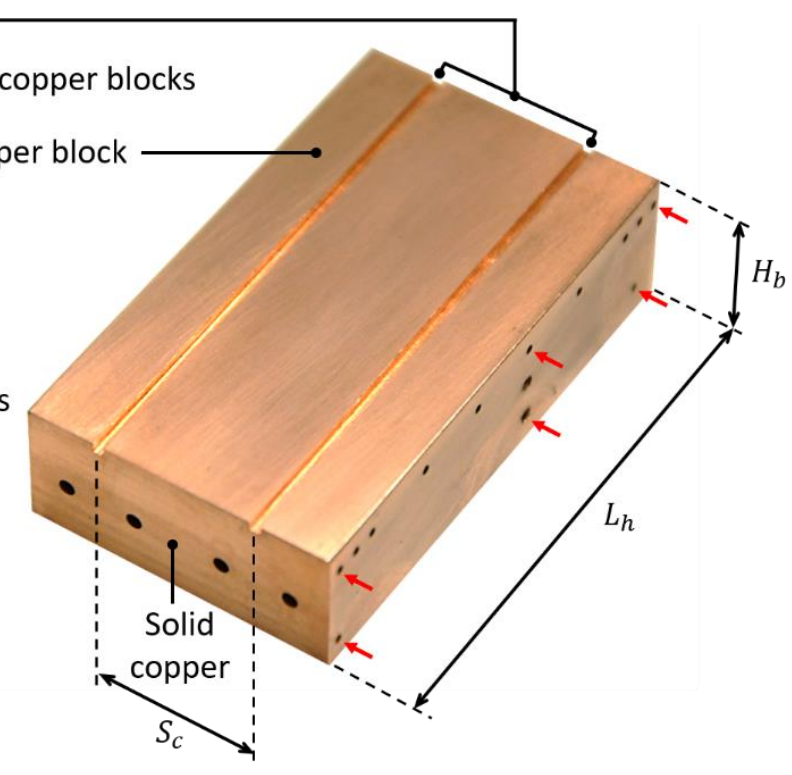

Thermally coupled channels

Fig. 3. Photograph of the channel blocks with key features and dimensions labelled: (a) for the thermally isolated case, individual copper blocks have an air gap maintained between them and (b) for the thermally coupled case, a single solid copper block is used. Thermocouple ports used for temperature measurements are indicated by red arrows. [2 columns]

\subsection{Sensor calibration and data reduction}

\subsubsection{Heat loss calibration}

A portion of the total power supplied to the test section is not absorbed by the fluid but is instead lost to the ambient. This power loss $P_{\text {loss }}$ is determined by draining water from the test section and then applying the power to each heater independently. Different power combinations are applied to span all possible combinations of the block temperatures that are experienced during the experiments. At each combination of applied power, the system is allowed to achieve a steadystate condition, where the temperature at any location on the block changes by less than $0.1^{\circ} \mathrm{C}$ over 
$0.5 \mathrm{~h}$. In this condition, all of the power supplied to the test section is lost to the ambient and the average block temperatures are recorded. Note that the thermally isolated configuration has two separate blocks, and therefore, each block will have different average temperature values, $T_{1}$ and $T_{2}$, when they have different powers applied to them. In contrast, the thermally coupled configuration has a single block and it attains a single, near-uniform temperature $\left(T_{1}=T_{2}=T\right)$ due to lateral wall conduction. A best-fit surface $\left(R^{2}=0.99\right)$ to the temperature data gives the equation for determining the power loss. For thermally isolated case it is given by $P_{\text {loss }, 1}(\mathrm{~W})=$ $0.092 T_{1}\left({ }^{\circ} \mathrm{C}\right)-0.049 T_{2}\left({ }^{\circ} \mathrm{C}\right)-1.16$. Note that the power loss for channel 2 can be calculated simply by swapping $T_{1}$ and $T_{2}$ as the channel blocks are identical.For the thermally coupled case it is given by $P_{\text {loss }}(W)=0.088 T\left({ }^{\circ} \mathrm{C}\right)-2.018$. The equation for the isolated case confirms a high degree of thermal isolation between channels as power loss from a individual channel has strong dependence on its own temperature and a weak dependence on the temperature of the other channel. However, in the equation for the coupled case, the power loss is observed to be a function of a single, uniform temperature value $\left(T=T_{1}=T_{2}\right)$ that is attained by walls of both the channels and the single copper block, thus indicating a strong thermal coupling.

The actual heating power being absorbed by the fluid flowing inside each channel is calculated by subtracting the power loss from the total electric power supplied using $P_{\text {in }}=P-P_{\text {loss }}$. At a given test condition, the same power $P$ is supplied to each channel block such that total power supplied to the test section is $P_{T}=2 P$. However, depending on the temperatures of the channels, $P_{\text {loss }}$ may be different for each channel, resulting in a different $P_{i n}$. The heat flux into the fluid is calculated using $q_{\text {in }}^{\prime \prime}=P_{\text {in }} / A_{\text {wall }}$, where $A_{\text {wall }}=2 H_{c} L_{h}+W_{c}$ is the wetted area of the channel walls. 


\subsubsection{Differential pressure transducer calibration}

The approach for calibrating the differential pressure transducers and measuring the flow rate in each channel is adopted from our previous study [24]. The key details of this approach are summarized here. For flow rate measurement in each channel, the current output from the differential pressure transducers is converted to a flow rate via a calibration against the liquid flow meter that measures the total flow rate $Q_{T}\left(=Q_{1}+Q_{2}\right)$. Fluid entering the test section as singlephase liquid is preheated to a fixed value of $88.5^{\circ} \mathrm{C}$ (the same as in the experiments) and the flow rate is increased in steps from 1 to $25 \mathrm{ml} / \mathrm{min}$. For the single-phase liquid, the total flow is divided equally between the channels by the inlet plenum. The characteristic flow rate versus current output curves are obtained for both the transducers, which are found to be identical. Therefore a single, combined linear fit is used to convert the measured signal to the channel flow rate for both the differential pressure transducers. This linear fit is given by $Q_{i}=2.108 I_{i}-8.433$, where $Q_{i}$ is the flow rate in $\mathrm{ml} / \mathrm{min}$ and $I_{i}$ is the measured transducer output current in milliamperes $(\mathrm{mA})$ for a given channel. The coefficient of determination for this linear fit is $R^{2} \approx 1$. For all tests, the flow distribution is represented as the fraction of the total flow rate going into each individual channel $\varepsilon_{i}=Q_{i} / Q_{T}$ such that the sum of the flow rate fractions is unity, i.e., $\varepsilon_{1}+\varepsilon_{2}=1$. The flow is uniformly distributed when $\varepsilon_{i}=0.5$ and maldistributed otherwise. In the maldistributed state one channel receives excess flow (e.g., channel 1 with $\varepsilon_{1}>0.5$ ) while the other channel is starved of the flow (channel 2 with $\varepsilon_{2}<0.5$ ).

To determine the flow distribution in the experiments, the flow rate is measured for the channel that is in the single-phase liquid regime (say channel 1 with a higher flow rate $Q_{1}$ ). The flow rate in the boiling channel (channel 2 with lower flow rate $Q_{2}$ in this case) is then calculated by subtracting the channel 1 flow rate from the total flow rate $\left(Q_{2}=Q_{T}-Q_{1}\right.$, where the total flow 
rate $Q_{T}$ is obtained from the liquid flow meter located upstream of the test section). The overall pressure drop across the channels is determined using the manufacturer-supplied calibration of the differential pressure transducer.

\section{Results and discussion}

This section presents a comparison of the flow distribution behavior of a two-channel system in thermally isolated versus thermally coupled configurations. The effect of thermal coupling on the flow distribution is analyzed as a function of increasing input power through synchronized measurements of flow rate in each channel, wall temperature difference, and heat dissipated into each channel. These results allow the key differences between the thermally isolated and coupled configurations to be observed and interpreted, specifically, in terms of the range of input powers with maldistributed flow, the severity of flow maldistribution, and the heat flux distribution between channels. This is followed by a comparison of the experimental results with the predictions from our two-phase flow distribution model.

The experimental procedure for characterizing the flow maldistribution is identical to that reported in our previous study [24]. Experiments are conducted at a single mass flow rate of $\sim 10$ $\mathrm{ml} / \mathrm{min}$. DI water is circulated through the flow loop at this constant flow rate and preheated to $\sim 88.5^{\circ} \mathrm{C}$, which corresponds to an inlet subcooling of $\sim 11.6^{\circ} \mathrm{C}$ based on the test section outlet pressure $(104.4 \mathrm{kPa})$. The flow rate and the inlet sub-cooling are maintained constant throughout the tests. The channels in both the thermally isolated and coupled configurations are heated uniformly and subjected to the same power. To study the effect of increasing total power on the flow maldistribution, the power input is increased in steps from $2.6 \mathrm{~W}$ to $17 \mathrm{~W}$, and the flow distribution is measured at each power level. This input power is the power absorbed by the fluid 
flowing in the channel and is obtained after accounting for heat loss to the ambient i.e., by subtracting the power loss to the ambient from the power applied to the heaters.

\subsection{Flow distribution behavior of thermally isolated versus thermally coupled channels}

Fig. 4. shows the (a) relative flow rate distribution, (b) wall temperature, and (c) heat flux for each of the two parallel channels as a function of the total input power. The thermally isolated case is shown on the left and the thermally coupled case on the right. Three regions are observed with increasing power in both configurations: in region (I), the flow in both channels is singlephase liquid and they receive equal flow rates; in region (II), boiling is observed in one channel while the other channel remains in a single-phase liquid flow regime and they receive unequal flow rates, i.e., flow maldistribution exists; in region (III) both the channels undergo boiling and they again receive equal flow rates. The following paragraphs will further discuss the behavior in each region, with emphasis on comparing and contrasting the thermally isolated versus coupled cases. Note that we have arbitrarily designated channel 1 as having the higher flow rate and channel 2 as having the lower flow rate in Fig. 4. However, from test to test, boiling can first occur in either channel, which would then receive the lower flow rate.

In region (I), from $P_{T, i n}=0-5 \mathrm{~W}$, it is clear that the behavior is not impacted by thermal coupling. Single-phase liquid flow is observed in both the channels and the total flow is evenly distributed between the channels by the inlet plenum, i.e., $\varepsilon_{i}=0.5$ (Fig. 4a1 and Fig. 4a2). Under single-phase and uniform flow conditions, the wall temperatures (Fig. 4b) of the two channels are equal (within measurement uncertainty) and increase linearly with increasing power input as expected. The channels receive the same heat flux (Fig. 4c1 and Fig. 4c2) and thus share the total heat load equally. 

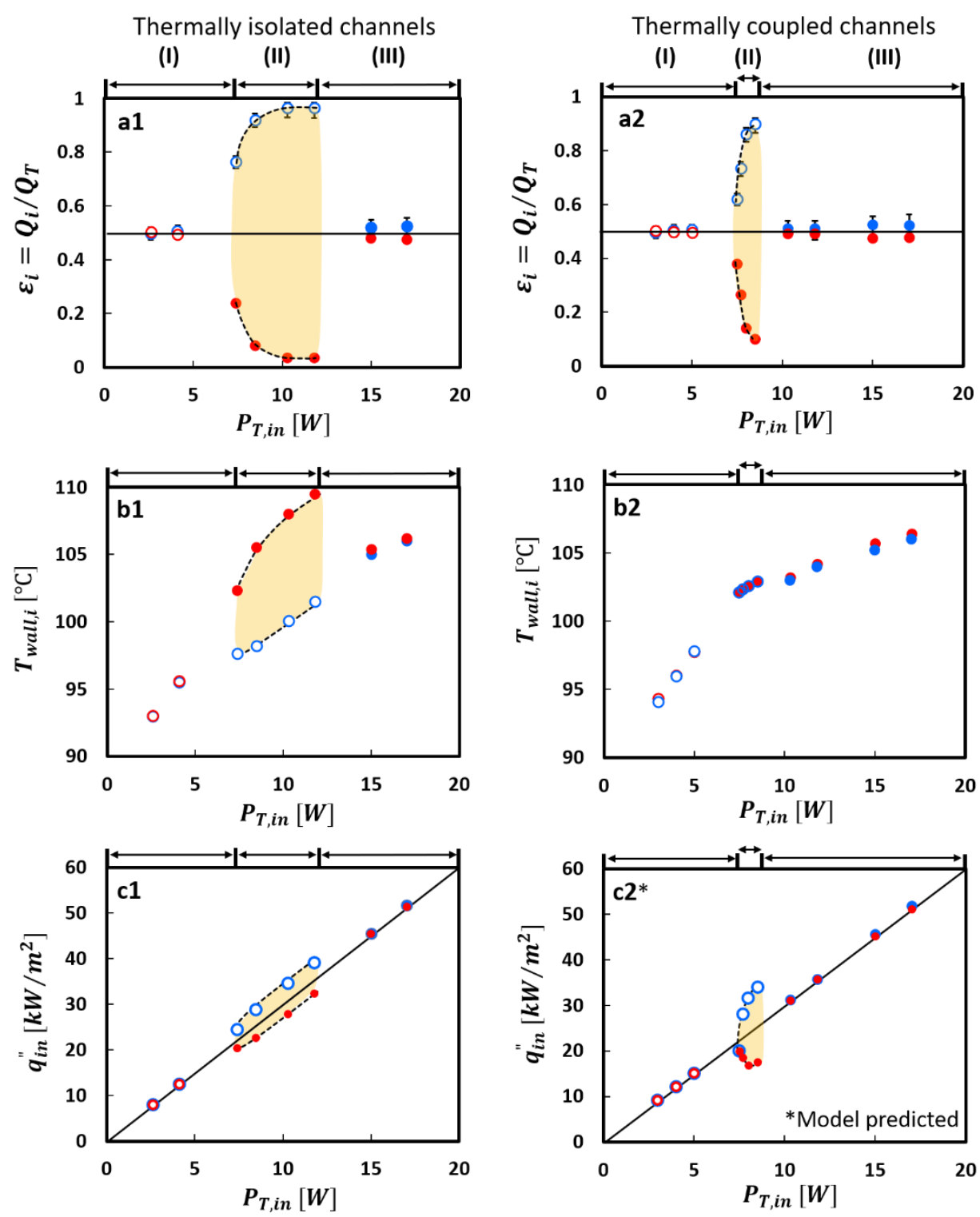

\begin{tabular}{l|l}
\hline Channel 1 & O / O Single-phase flow \\
\hline
\end{tabular}

Channel 2 Two-phase flow

Fig. 4. Comparison of the thermal and hydrodynamic characteristics of thermally isolated (left) and thermally coupled (right) cases: (a) fraction of the total flow rate going into each channel, (b) channel wall temperature, and (c) heat flux into the fluid, all as a function of total input power. The black horizontal line in (a) represents an even flow distribution between the channels. The black diagonal line in (c) represents an equal heat flux going into both channels. The flow regime in each channel is denoted by marker type: open circles $(O)$ for single-phase liquid flow and filled 
circles ( ) for two-phase flow. Three labeled regions indicate the operating conditions where: (I) flow through both the channels is single-phase, (II) there is single-phase flow in channel 1 and boiling in channel 2, and (III) boiling occurs in both the channels. Note that the data reported in Fig. c2 is obtained from the model because the thermally coupled channels attain the same temperature as that of the channel block, and therefore it is not possible to determine the heat loss separately for each channel from the experimental data. [1.5 columns]

At a total input power of $P_{T, i n}=7.4 \mathrm{~W}$, the start of region II, boiling is observed in channel 2 while channel 1 remains in the single-phase liquid regime (in both configurations). Once boiling incipience occurs in channel 2, the Ledinegg instability is triggered and causes non-uniform flow distribution between the two channels (i.e., more flow through channel 1 than channel 2) and the severity of flow maldistribution increases with increasing power. A detailed discussion of this transient excursion event at the onset of boiling is provided in our previous work for the thermally isolated case [24]. The range of total input power over which flow maldistribution exists between the channels defines region II (shaded yellow in Fig. 4) for both configurations. There are clear differences between the thermally isolated and thermally coupled configurations within this region of maldistributed flow.

In thermally isolated channels, flow maldistribution occurs over the total input power range, $P_{T, i n}$, of $7.4 \mathrm{~W}$ to $11.8 \mathrm{~W}$. This range is noticeably narrower for the thermally coupled channels, which goes from $7.4 \mathrm{~W}$ to $8.5 \mathrm{~W}$. Further, the maximum severity of flow maldistribution is lesser in the thermally coupled case. Specifically, the flow rate fraction in the thermally isolated case can be as low as $\varepsilon_{i} \cong 3.5 \%$ (at $P_{T, \text { in }}=11.8 \mathrm{~W}$ ) in the starved channel, compared to a minimum of $\varepsilon_{i} \cong 10 \%$ (at $P_{T, i n}=8.5 \mathrm{~W}$ ) in the thermally coupled case. The deleterious effect of the 
maldistribution on the wall temperature is also much more severe in the isolated case. Maldistribution causes the flow-starved channel to have a large increase in temperature (from $T_{2}=$ $102.3^{\circ} \mathrm{C}$ at $P_{T, \text { in }}=7.4 \mathrm{~W}$ to $T_{2}=109.5^{\circ} \mathrm{C}$ at $P_{T, \text { in }}=11.8 \mathrm{~W}$ ) and increasing maldistribution with power induces an increase in the wall temperature difference between the channels (as seen in Fig. 4b1). The wall temperature of channel 1 remains at a lower temperature, with an increase from $T_{1}=$ $97.6^{\circ} \mathrm{C}$ at $P_{T, \text { in }}=7.4 \mathrm{~W}$ to $T_{1}=101.2^{\circ} \mathrm{C}$ at $P_{T, \text { in }}=11.8 \mathrm{~W}$; this behavior is commensurate with a large increase in the flow rate of $\sim 20 \%$ initially, followed by single-phase operation at higher input powers.

In stark contrast with the large wall temperature difference observed for the thermally isolated channel, the thermally coupled case sees the wall temperatures of both channels remain nearly equal throughout region II despite the flow maldistribution. Further, this wall temperature value of $\sim 102.5^{\circ} \mathrm{C}$ is much lower than the maximum wall temperature of $\sim 109.5^{\circ} \mathrm{C}$ observed in the thermally isolated case. This clearly demonstrates that thermal coupling reduces the severity of flow maldistribution, wall temperature difference between the channels, and maximum wall temperature of the flow-starved channel.

The different behavior exhibited by the thermally isolated and coupled channels can be attributed to the mechanism of heat redistribution between the two channels. In the isolated case, once flow maldistribution occurs at $P_{T, \text { in }}=7.4 \mathrm{~W}$, the heat flux into both the channels increases linearly with increasing power (Fig. 4c1) and, at any given power level, the two channels receive approximately the same heat flux. Slight differences between the heat flux values and the black diagonal line (denoting an equal heat flux into each channel) are due to the differences in power loss caused by the large difference in wall temperatures. Nevertheless, even when the flow rate between the two channels is severely maldistributed, each channel must independently dissipate 
its share of the total heat load to the coolant, as no channel-to-channel heat exchange is possible. In comparison, strong thermal coupling between the channels by lateral heat conduction, indicated by the isothermal block temperature, allows for redistribution of the total input heat load and large differences in the heat flux going into each channel. For example, in the coupled case (Fig. 4c2), once flow maldistribution is triggered at $P_{T, i n}=7.4 \mathrm{~W}$, the heat flux in channel 1 increases with increasing power, while the heat flux decreases in channel 2. In other words, channel 1 tends to dissipate an increasingly larger share of the heat load as the flow maldistribution worsens with increasing power. Under the most severe flow maldistribution (at $P_{T, \text { in }}=8.5 \mathrm{~W}$ ), channel 1 receives $\sim 90 \%$ of total flow rate and dissipates $\sim 66 \%$ of $P_{T, i n}$, while channel 2 dissipates the remaining $34 \%$.

At some maximum total power input in region (II), the wall temperature of channel 1 becomes large enough to trigger boiling incipience, marking the transition to region (III), as shown in Fig. 4 where both channels experience boiling. For the tested power levels, this occurred at $P_{T, \text { in }}$ $=15 \mathrm{~W}$ in the thermally isolated case and at $P_{T, i n}=10.3 \mathrm{~W}$ in the coupled case. For the isolated case, the onset of boiling in channel 1 caused an associated reduction in the wall temperature of channel 2 from $T_{2}=109.5^{\circ} \mathrm{C}$ at $P_{T, \text { in }}=11.8 \mathrm{~W}$ to $T_{2}=104^{\circ} \mathrm{C}$ at $P_{T, i n}=15 \mathrm{~W}$ (Fig. $4 \mathrm{~b} 1$ ), a result of the dramatic increase in flow rate relative to a uniform flow distribution (Fig. 4a1). For the thermally coupled case, the reduction in the wall temperature of channel 2 at the transition to region (III) is less dramatic because, for reasons discussed above, its temperature was not elevated due to maldistribution in region (II). Following the transition to region (III), the thermally isolated and coupled cases follow a similar trend. The flow resistances of the two channels become approximately equal again once they are boiling and the flow maldistribution caused by the 
Ledinegg instability is suppressed. The heat fluxes to each channel are again equal at each power level (Fig. 4c1 and Fig. 4c2) and they attain the same temperature (Fig. 4b1 and Fig. 4b2).

The flow in both microchannels was visualized at each power level to corroborate the flow regimes inferred for each channel in the discussion above. Fig. 5 shows selected flow visualization and an accompanying schematic for the case of thermally coupled parallel microchannels. The flow direction is from left to right and the entire heated length of the channels is shown in the images. The flow rate to each channel in each of the three regimes is qualitatively represented by the length of the arrows near the channel inlets. The flow visualizations captured at each power level enable the two-phase morphology to be identified and support the trends shown in Fig. 4. That is, different regions of operation exist, where (I) both channels are in the single-phase flow regime, (II) boiling is observed in channel 1 and single-phase flow is observed in channel 2, or (III) both channels undergo boiling. It clear from Fig. 5 that the flow is evenly distributed between the channels when they both are in the single-phase liquid regime (I) or they both undergo boiling (III), while the flow is maldistributed when only one of the channels undergoes boiling (regime II). In regime II, the flow maldistribution increases with increasing input power. 
(I) Single-phase liquid flow in both channels

\section{Channel 1}
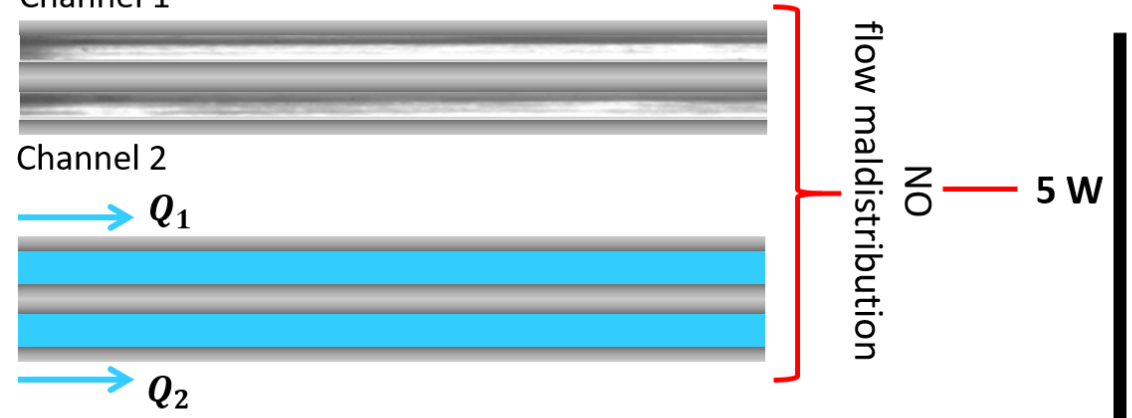

(II) Single-phase liquid flow in channel 1

Two-phase flow in channel 2
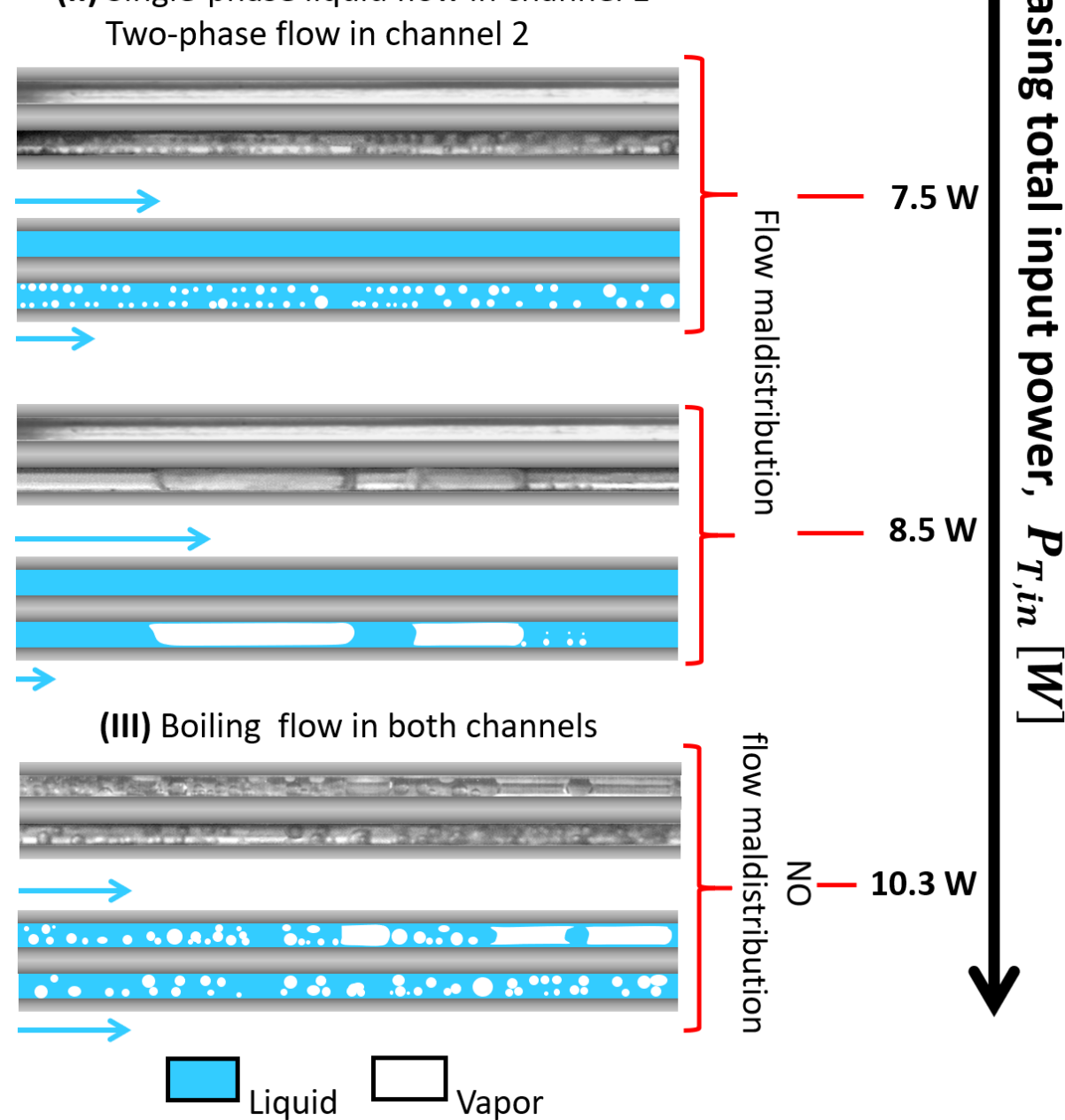

Fig. 5. Flow visualization images and accompanying schematic representations of the flow regimes observed in each channel: (I) single-phase liquid flow in both channels, (II) boiling in channel 2 and single-phase flow in channel 1, and (III) boiling in both the channels. Note that these characteristic regimes were the same for both thermally isolated and coupled cases; the 
representative images above are extracted from the thermally coupled case visualizations. The flow direction is from left to right. [1.5 columns]

\subsection{Pressure drop characteristics of thermally isolated versus thermally coupled channels}

Even though the two parallel microchannels can be thermally isolated or coupled, recall that they are hydrodynamically coupled via the same inlet and outlet plenums, and therefore, have a common pressure drop. Even as boiling occurs in one or both of the channels, and the overall pressure drop across them increases (due to an increase in flow resistance), the pressure drop remains the same across both the channels. This is the mechanism by which the flow rate in each individual channel readjusts to satisfy the constant total flow rate boundary condition that is set by the pump. The stable, maldistributed flows described above are possible due to the non-monotonic nature of the channel demand curve, which allows flow rates through the channels to be drastically different at the same pressure drop [20, 30-32]. We emphasize here that the system boundary conditions in the current experiments (uniform pressure drop across the channels at a constant total flow rate) are the same as would be encountered by individual channels in a heat sink having an array of multiple parallel channels.

With this understanding, the flow resistance characteristics of the two parallel channels, in the thermally isolated and the coupled configurations, are compared in Fig. 6, which shows the pressure drop across the channels as a function of $P_{T, i n}$. Both the isolated and coupled configurations exhibit the same general trend of increasing pressure drop with increasing power, as would be expected. In region (I), when flow through both channels is single-phase liquid and uniformly distributed, the pressure drop remains fairly constant at $\sim 81 \mathrm{~Pa}$ for both configurations. 


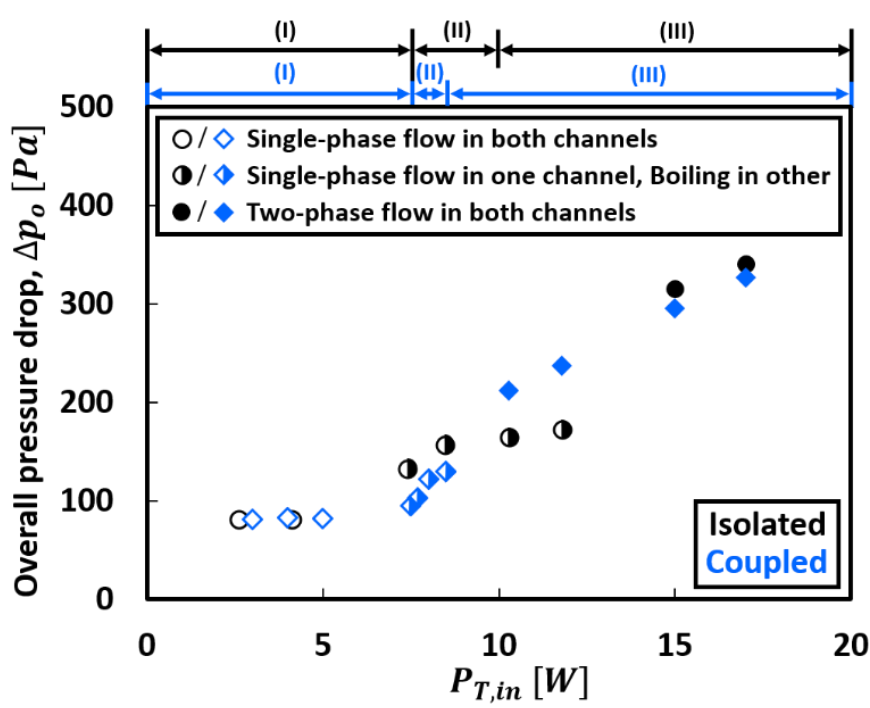

Fig. 6. Pressure drop across the two parallel channels as a function of the total input power for both thermally isolated and coupled configuration. Open markers $(O, \diamond)$ denote single-phase flow in both channels; half-filled markers $(\boldsymbol{\top}, \triangleleft)$ denote single-phase flow in channel 1 and two-phase flow in channel 2; and solid markers $(\bullet, \bullet)$ denote two-phase flow in both the channels. [1 column]

Once boiling occurs in channel 2 at $P_{T, i n}=7.4 \mathrm{~W}$, the flow resistance increases due to the vapor generation and increases the overall pressure drop within region (II) to $\sim 132 \mathrm{~Pa}$ in the isolated case and $\sim 95 \mathrm{~Pa}$ in the coupled case. With further increases in power within region (II) there is a moderate increase in pressure drop, from $\sim 132 \mathrm{~Pa}\left(P_{T, \text { in }}=7.4 \mathrm{~W}\right)$ to $\sim 170 \mathrm{~Pa}\left(P_{T, \text { in }}=\right.$ $11.8 \mathrm{~W})$ in the isolated case and from $\sim 95 \mathrm{~Pa}$ at $\left(P_{T, i n}=7.4 \mathrm{~W}\right)$ to $\sim 125 \mathrm{~Pa}\left(P_{T, i n}=8.5 \mathrm{~W}\right)$ in the coupled case. This pressure drop is lower in the coupled case compared to the isolated case due to the lower vapor quality in channel 2 . This lower vapor quality is a combined result of less severe flow maldistribution and lower heat flux into the boiling channel (channel 2) due to the redistribution of heat flux. For a representative case of $P_{T, \text { in }}=7.4 \mathrm{~W}$, the vapor quality in channel 2 in the isolated case is $x_{\text {out }} \sim 0.02$, which is an order of magnitude higher compared to that of the 
coupled case ( $\left.x_{\text {out }} \sim 0.004\right)$. Therefore, thermal coupling, by mitigating the flow distribution, also can significantly reduce the pressure drop in this region. The vapor quality is calculated as $x_{\text {out }}=$ $\frac{P_{i n}-\dot{m} c_{p}\left(T_{f l, o u t}-T_{f l, i n}\right)}{\dot{m} h_{f g}}$, where, $x_{\text {out }}$ is the vapor fraction at the exit, $P_{i n}$ is the power absorbed by the channel, $T_{f l, i n}$ is the fluid inlet temperature, $T_{f l, o u t}$ is the fluid outlet temperature, and $\dot{m}$ is the mass flow rate in the channel. In both the cases, the additional flow resistance caused by boiling in channel 2 increases the overall pressure drop from region (I) to region (II). However, throughout region (II) an increase in input power does not increase the overall pressure drop drastically because with more vapor generation in the starved channel the fluid is rerouted to channel 1 , which is still in the single-phase regime.

When boiling starts occurring in both channels at $P_{T, i n}=15 \mathrm{~W}$ in the isolated case and at $P_{T, \text { in }}=10.3 \mathrm{~W}$ in the coupled case, the pressure drop increases significantly to $\sim 212 \mathrm{~Pa}$ and $\sim 315$ $\mathrm{Pa}$, respectively. This corresponds to the beginning of region (III) as shown in Fig. 6. Note that this higher pressure drop in the isolated case compared to the coupled case is merely due to boiling in both the channels occurring at a higher input power in the latter case. Otherwise, throughout region (III), the pressure drop increases with increasing total power input for both cases (due to the increased flow resistance associated with vapor generation within both the channels) and is approximately the same at a given power. In summary, the overall pressure drop characteristics of thermally isolated and coupled channels are identical when flow is evenly distributed between the channels and they are either in a single-phase liquid regime (region I) or boiling (region III). However, under maldistributed flow conditions (region II), thermal coupling is more effective in reducing pressure drop compared to the isolated case. 


\subsection{Comparison of experimental results with model predictions}

In this section, the experimental data are compared with predictions made using a twophase flow distribution model that accounts for channel-to-channel thermal coupling. Our modeling approach is described in detail in Ref. [30] and is implemented in an identical manner here. To summarize, the approach predicts the stable flow rate distributions in a system of multiple parallel heated microchannels for a subcooled inlet liquid flow. The methodology couples a thermal-hydraulic model for individual channels (load curve) with the pump curve in a system of flow network equations. The heat transfer accounts for the internal convection in the channels, heat loss to the ambient, and axial and lateral thermal conduction in the solid walls. Because lateral thermal conduction plays a critical role in the flow distribution behavior between parallel channels, it is incorporated in the model through a thermal conductance, $C_{\text {lat }}$, that quantifies the degree of thermal coupling between the channels. The thermal conductance is defined based on onedimensional heat conduction between the vertical mid-planes of the channels as $C_{\text {lat }} \cong k H_{b} L_{c} / S_{c}$. A value of $C_{\text {lat }}=0 \mathrm{~W} / \mathrm{K}$ indicates perfect thermal isolation between the channels and an increasing conductance signifies increasing thermal coupling.

To compare the model-predicted flow distributions with the experimental data, the experimental parameters and operating conditions listed in Table 1 are used as inputs to the model. For the thermally coupled configuration, the lateral thermal conductance is calculated as $C_{\text {lat }} \cong$ $k H_{b} L_{c} / S_{c}=18.5 \mathrm{~W} / \mathrm{K}$ using the properties of the solid copper block because it offers the leastresistance path for heat flow between the channels. This is representative of the typical extent of thermal coupling that would be present in microchannel heat sinks with many parallel channels. For the thermally isolated configuration, $C_{\text {lat }}$ cannot be simply determined based on heat conduction through the air gap because heat primarily conducts through the polycarbonate cover 
and ceramic base of the test section. Instead, the model predictions are first validated against the experiments for the thermally coupled configuration, and then $C_{\text {lat }}$ is calibrated to the experimental data for the thermally isolated configuration. By varying the input thermal conductance to the model, a single value of $C_{\text {lat }} \cong 0.36 \mathrm{~W} / \mathrm{K}$ is found to best match the measured flow maldistribution (within experimental bounds) across all tested power levels for the thermally isolated configuration. For $C_{\text {lat }} \cong 0.36 \mathrm{~W} / \mathrm{K}$ the mean absolute percentage error (MAPE) between the experimentally measured and the model predicted flow rate in channel 1 (channel with excess flow) is within $3.5 \%$ for any tested power level. This small value of $C_{\text {lat }}$ confirms that the air gap in the experimental setup maintains a high degree of thermal isolation between the channels. The axial cross-section area of each channel block $A_{b}=\left(H_{b} \times W_{b}\right)-\left(H_{c} \times W_{c}\right)$ is used to model axial wall conduction. Heat loss to the ambient is neglected, and therefore, $\frac{P_{T, i n}}{2}$ is considered as the power going into each channel in the model.

Table 1. Parameters from the experiments used as inputs to the flow distribution model [30]. Fluid properties are taken for DI water at $88.5^{\circ} \mathrm{C}$. [1 column]

\begin{tabular}{lc}
\hline Parameter (symbol) & Value \\
\hline Channel height $\left(H_{c}\right)$ & $1 \mathrm{~mm}$ \\
Channel width $\left(W_{c}\right)$ & $1 \mathrm{~mm}$ \\
Channel length $\left(L_{c}\right)$ & $55 \mathrm{~mm}$ \\
Channel pitch $\left(S_{c}\right)$ & $16 \mathrm{~mm}$ \\
Inlet mass flux $(G)$ & $150 \mathrm{~kg} / \mathrm{m}^{2}-\mathrm{s}$ \\
Fluid inlet temperature $\left(T_{f l, i n}\right)$ & $88.5^{\circ} \mathrm{C}$ \\
Outlet pressure $\left(p_{\text {out }}\right)$ & $104.4 \mathrm{kPa}$ \\
Total input power $\left(P_{T, \text { in }}\right)$ & $2.6-17 \mathrm{~W}$
\end{tabular}


Thermal conductivity of channel wall $(k)$

Lateral thermal conductance

$\left(C_{\text {lat }}\right)$

Thermally isolated

Thermally coupled

Channel block height $\left(H_{b}\right)$

Channel block width $\left(W_{b}\right)$

Channel block length $\left(L_{b}\right)$
$360 \mathrm{~W} / \mathrm{m}-\mathrm{K}$

$0.36 \mathrm{~W} / \mathrm{K}^{\dagger}$

$18.5 \mathrm{~W} / \mathrm{K}^{*}$

$15 \mathrm{~mm}$

$15 \mathrm{~mm}$

$55 \mathrm{~mm}$

tCalibrated using the model as described in the text

${ }^{*}$ Calculated as $C_{\text {lat }}=\frac{k H_{b} L_{c}}{S_{c}}$

The comparison of the experimental and modeling results is presented in Fig. 7, which shows the flow rate fraction in each channel versus the total input power for the thermally isolated and coupled cases. In both cases, the model is able to accurately capture all of the critical features of the experiments. The total flow rate is evenly distributed between the channels when they both are in the single-phase liquid flow regime (I). At power levels where boiling occurs in only one of the channels, the flow resistance in that channel increases and triggers severe flow maldistribution via the Ledinegg instability. Excellent quantitative agreement is again observed between the experimental measurements and model predictions. For the thermally coupled case for which all model inputs are known a priori, the mean absolute percentage error (MAPE) in the channel 1 flow rate is $7.2 \%$ over the range of power levels where flow maldistribution occurs. Some discrepancy is expected due to the accuracy bounds of the two-phase pressure drop and the heat transfer coefficient correlations used in the model. 

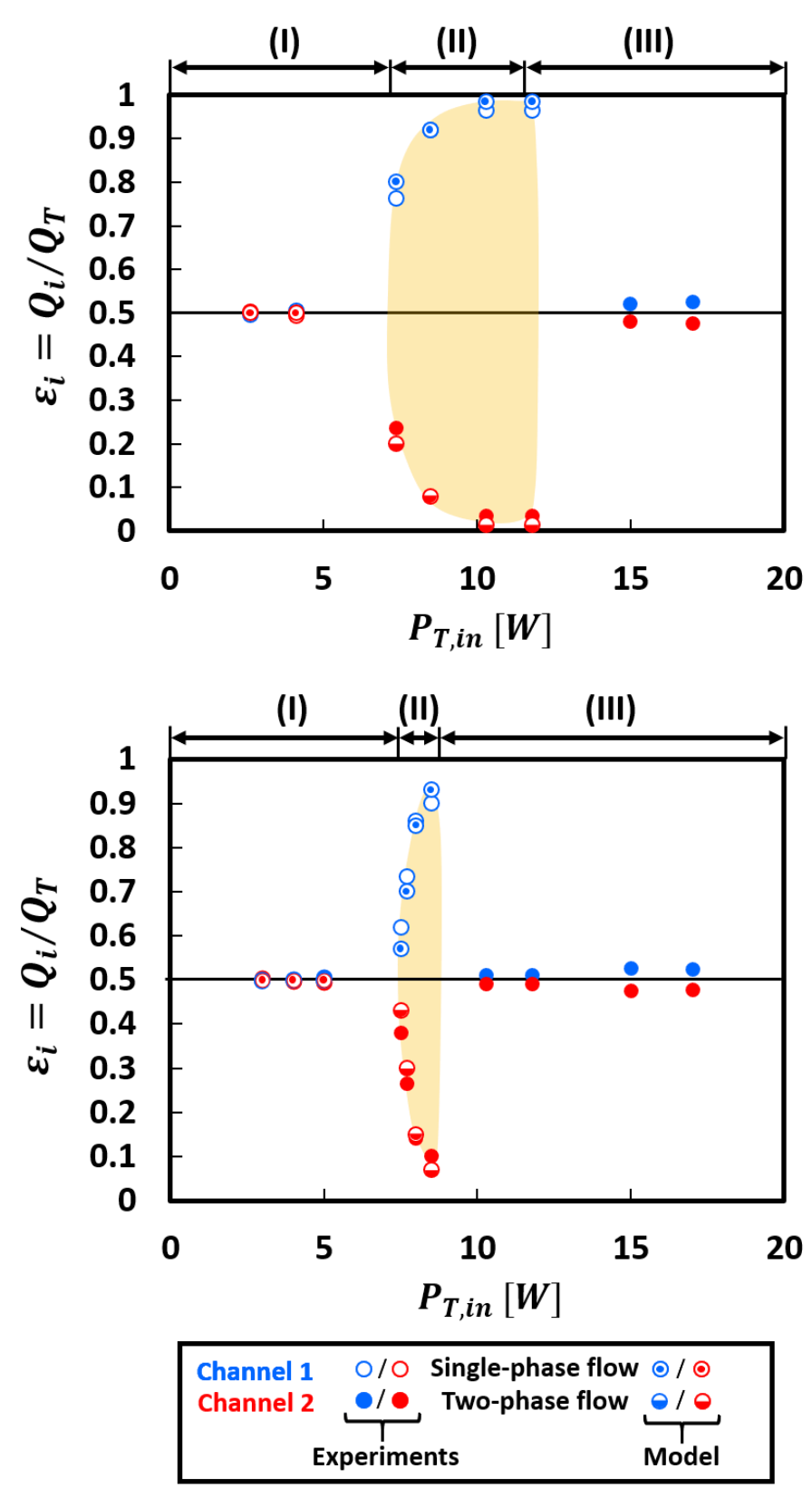

Fig. 7. Comparison of flow rate distribution between the current experiments and model predictions (input parameters given in Table 1) in the (top) thermally isolated configuration and (bottom) thermally coupled configuration. The flow regime in each channel is denoted by marker type. For experimental results: open circular markers $(O)$ for single-phase liquid flow and solid circles (๑) for two-phase flow. For modeling results: circular dots ( $(0)$ for single-phase liquid flow and half-filled circles $(\Theta)$ for two-phase flow. Three labeled regions indicate the operating 
conditions where: (I) flow through both the channels is single-phase, (II) there is single-phase flow in channel 1 and boiling in channel 2, and (III) boiling occurs in both the channels. [1 column]

This agreement between the model predictions and experiments firmly establishes the primary conclusions of this work regarding the influence of lateral wall conduction on the flow distribution behavior. The flow maldistribution between the two channels is mitigated in the thermally coupled case, both in terms of the maximum severity of flow maldistribution and the range of total input power that lead to maldistribution, as shown quantitatively in Table 2. A strong match between the behavioral trends observed in experiments (Fig. 4a1 and Fig. 4a2) and obtained via model predictions (Fig. 7) confirms the effect of thermal coupling, primarily, that a strong thermal coupling leads to a more uniform flow distribution.

Table 2. A comparison of isolated versus coupled case based on the flow distribution parameters.

\begin{tabular}{cccccr}
\hline & Thermally isolated & \multicolumn{2}{l}{ Thermally coupled } \\
\hline Parameters & Experiments & Model & Experiments & Model \\
\hline $\begin{array}{c}\text { Maximum severity of flow maldistribution } \\
\text { (\% of total flow rate in flow starved channel) }\end{array}$ & 3.5 & 1.5 & 10 & 7.5 \\
$\begin{array}{c}\text { Range of input power with maldistributed } \\
\text { flow (W) }\end{array}$ & $7.4-11.8$ & - & $7.4-8.5$ & - \\
\hline
\end{tabular}

\section{Conclusions}

Existing two-phase microchannel heat sink design efforts have been typically restricted to models that assume uniform flow. In the few approaches that have considered the flow distribution, 
models still do not incorporate the effects of thermal coupling between the parallel channels. Despite recent theoretical investigations that have pointed toward the importance of thermal coupling, no experimental data on the flow distribution in boiling parallel microchannels has been reported to verify and validate this behavior.

This study experimentally investigates, and theoretically verifies, the effect of channel-tochannel thermal coupling on the flow maldistribution caused by the Ledinegg instability in a system with two parallel microchannels. Deionized water is delivered at a constant total flow rate to the channels that share common inlet and outlet plenums, and therefore have the same pressure drop. The channels are uniformly heated and subjected to the same power level, which is increased in steps. Two configurations are investigated, namely, thermally isolated and thermally coupled, that have two orders of magnitude difference in the lateral thermal conductance between the channels, with otherwise identical channel parameters. The flow rate in each channel is directly measured simultaneously with the wall temperature and overall pressure drop across the channels, which allows for differentiation between the thermal and hydrodynamic behaviors of these two configurations.

Thermal coupling is observed to play a significant role in mitigating flow maldistribution in terms of reducing the maximum severity of flow maldistribution between channels and narrowing the range of input power over which the flow maldistribution occurs. In the most severely maldistributed state, the starved channel receives just $3.5 \%$ of the total flow in the thermally isolated configuration versus $10 \%$ in the thermally coupled configuration. This is because the channel-to-channel thermal coupling allows redistribution of the heat input from the flow-starved channel to the neighboring channel. This heat load redistribution also allows the thermally coupled channels to attain a near-equal wall temperature irrespective of the severity of 
flow maldistribution, with a much lower maximum wall temperature at the most severe flow maldistribution condition. In contrast, in the thermal isolated channels, the flow-starved channel must continue to independently dissipate its share of the heat load, which causes a large wall temperature difference between the channels to grow with increasing power, due to the worsening flow maldistribution. A direct comparison drawn between the experimental data and predictions from a two-phase flow distribution model is found to have strong agreement, thereby confirming the mitigating influence of lateral thermal coupling on flow maldistribution (resulting from the Ledinegg instability). Lateral thermal conduction between the channels leads to a more uniform flow distribution and wall temperature between the channels, which benefits the performance of microchannel heat sinks where the neighboring channels are thermally coupled via a shared substrate.

\section{Acknowledgments}

This material is based upon work supported by Ford Motor Company through the University Research Program (URP). Special thanks to Dr. Edward Jih at Ford Research \& Advanced Engineering (R\&AE) for technical discussions related to this work. The first author would like to acknowledge the Science and Engineering Research Board (SERB) and Indo-US

Science and Technology Forum (IUSSTF) for support through a SERB Indo-US Postdoctoral Fellowship. 


\section{References}

[1] T.G. Karayiannis, M.M. Mahmoud, Flow boiling in microchannels: Fundamentals and applications, Applied Thermal Engineering, 115 (2017) 1372-1397.

[2] J.B. Marcinichen, J.A. Olivier, J.R. Thome. On-chip two-phase cooling of datacenters: Cooling system and energy recovery evaluation. Applied Thermal Engineering, 41(2012) $36-51$.

[3] S. V. Garimella \& T. Harirchian. Encyclopedia of Thermal Packaging: Volume 1: Microchannel Heat Sinks for Electronics Cooling (2013).

[4] C. Park, J. Zuo, P. Rogers, J. Perez. Two-Phase flow cooling for vehicle thermal management. SAE Technical Paper (2005) 2005-01-1769.

[5] M.J. Mackowski. Requirements for high flux cooling of future avionics systems. SAE Technical Paper, (1991) 912104.

[6] Y. K. Prajapati \& P. Bhandari. Flow boiling instabilities in microchannels and their promising solutions-A review. Experimental Thermal and Fluid Science, 88 (2017)576593.

[7] A.E. Bergles, S.G. Kandlikar, On the nature of critical heat flux in microchannels, Journal of Heat Transfer, 127(1) (2005) 101-107.

[8] J.A. Boure, A.E. Bergles, L.S. Tong, Review of two-phase flow instability, Nuclear Engineering and Design, 25(2) (1973) 165-192.

[9] L.C. Ruspini, C.P. Marcel, A. Clausse, Two-phase flow instabilities: A review, International Journal of Heat and Mass Transfer, 71 (2014) 521-548.

[10] L. Tadrist, Review on two-phase flow instabilities in narrow spaces, International Journal of Heat and Fluid Flow, 28(1) (2007) 54-62.

[11] S. Kakac, B. Bon, A review of two-phase flow dynamic instabilities in tube boiling systems, International Journal of Heat and Mass Transfer, 51(3-4) (2008) 399-433. 
[12] E.R. Dario, L. Tadrist, J.C. Passos, Review on two-phase flow distribution in parallel channels with macro and micro hydraulic diameters: Main results, analysis, trends, Applied Thermal Engineering, 59(1-2) (2013) 316-335.

[13] M. Ledinegg, Instability of flow during natural and forced circulation, Die Warme, 61(8) (1938) 891-898.

[14] S.G. Kandlikar, W.K. Kuan, D.A. Willistein, J. Borrelli, Stabilization of flow boiling in microchannels using pressure drop elements and fabricated nucleation sites, Journal of Heat Transfer, 128(4) (2006) 389-396.

[15] A. Koşar, C.J. Kuo and Y. Peles, Suppression of boiling flow oscillations in parallel microchannels by inlet restrictors, Journal of Heat Transfer, 128(3) (2006) 251-260.

[16] B.A. Odom, M.J. Miner, C.A. Ortiz, J.A. Sherbeck, R.S. Prasher, P.E. Phelan, Microchannel two-phase flow oscillation control with an adjustable inlet orifice. Journal of Heat Transfer, 134(12), (2012) 122901.

[17] T. Zhang, J.T. Wen, A. Julius, H. Bai, Y. Peles, and M.K. Jensen, Parallel-channel flow instabilities and active control schemes in two-phase microchannel heat exchanger systems. In: Proceedings of the IEEE American Control Conference, June (2010) 37533758.

[18] Y. Taitel, U. Minzer, D. Barnea, A control procedure for the elimination of mal flow rate distribution in evaporating flow in parallel pipes. Solar Energy, 82(4) (2008) 329-335.

[19] C.J. Kuo, Y.Peles, Pressure effects on flow boiling instabilities in parallel microchannels, International Journal of Heat and Mass Transfer, 52(1-2) (2009) 271-280.

[20] K. Akagawa, M. Kono, T. Sakaguchi, M. Nishimura, Study on distribution of flow rates and flow stabilities in parallel long evaporators, Bulletin of Japan Society of Mechanical Engineers, 14(74) (1971) 837-848.

[21] U. Minzer, D. Barnea, Y. Taitel, Evaporation in parallel pipes - splitting characteristics, International Journal of Multiphase Flow, 30(7-8) (2004) 763-777. 
[22] U. Minzer, D. Barnea, Y. Taitel, Flow rate distribution in evaporating parallel pipes modeling and experimental, Chemical Engineering Science, 61(22) (2006) 7249-7259.

[23] T.A. Kingston, J.A. Weibel, S.V. Garimella, Ledinegg instability-induced temperature excursion between thermally isolated, heated parallel microchannels, International Journal of Heat and Mass Transfer, 132 (2019) 550-556.

[24] A. Miglani, J.A. Weibel, S.V. Garimella, Measurement of flow maldistribution induced by the Ledinegg instability during boiling in thermally isolated parallel microchannels, International Journal of Multiphase Flow, in review

[25] R.D. Flynn, D.W. Fogg, J.-M. Koo, C.-H. Cheng, K.E. Goodson, Boiling flow interaction between two parallel microchannels, In: Proceedings of ASME International Mechanical Engineering Congress and Exposition, Nov. 5-10, Chicago, Illinois, USA, 47853 (2006) 317-322.

[26] S. N. Ritchey, J. A. Weibel, S. V. Garimella, Local measurement of flow boiling heat transfer in an array of non-uniformly heated microchannels. International Journal of Heat and Mass Transfer, 71 (2014) 206-216.

[27] E. S. Cho, J. W. Choi, J. S. Yoon, M. S. Kim, Experimental study on microchannel heat sinks considering mass flow distribution with non-uniform heat flux conditions. International Journal of Heat and Mass Transfer, 53(9-10) (2010) 2159-2168.

[28] T. Zhang, J. T. Wen, A. Julius, Y. Peles, M. K. Jensen, Stability analysis and maldistribution control of two-phase flow in parallel evaporating channels. International Journal of Heat and Mass Transfer, 54(25-26) (2011) 5298-5305.

[29] R. Flynn, C.-H. Cheng, K. Goodson, Decoupled thermal and fluidic effects on hotspot cooling in a boiling flow microchannel heat sink, In: Proceedings of ASME International Technical Conference and Exhibition on Packaging and Integration of Electronic and Photonic Microsystem, July 8 - 7, Vancouver, British Columbia, Canada, 42770 (2007) 179-184. 
[30] T. Van Oevelen, J.A. Weibel, S.V. Garimella, The effect of lateral thermal coupling between parallel microchannels on two-phase flow distribution, International Journal of Heat and Mass Transfer, 124 (2018) 769-781.

[31] T. Van Oevelen, J.A. Weibel, S.V. Garimella, Predicting two-phase flow distribution and stability in systems with many parallel heated channels, International Journal of Heat and Mass Transfer, 107 (2017) 557-571.

[32] T. Zhang, T. Tong, J.Y. Chang, Y. Peles, R. Prasher, M.K. Jensen, J.T. Wen, P. Phelan, Ledinegg instability in microchannels, International Journal of Heat and Mass Transfer, 52(25-26) (2009) 5661-5674. 